\title{
Comparison of the Efficacy and Tolerability between Same-day Picosulfate and Split-dose Polyethylene Glycol Bowel Preparation for Afternoon Colonoscopy: A Prospective, Randomized, Investigator-blinded Trial
}

\author{
Mi Seon Kang, Tae Oh Kim, Eun Hee Seo, Da Kyung Jung, Mo Se Kim, Nae Yun Heo, Jong Ha Park, \\ Seung Ha Park, Young Soo Moon \\ Departments of Internal Medicine, Haeundae Paik Hospital, Inje University College of Medicine, Busan, Korea
}

Background/Aims: In the present study, we evaluated the efficacy and tolerability between same-day bowel preparation protocols using 2 sachets of Picosulfate and a $4 \mathrm{~L}$ split-dose polyethylene glycol (PEG) bowel preparation for afternoon colonoscopy. Methods: The study had a single-center, prospective, randomized, and investigator-blinded, non-inferiority design. We evaluated bowel preparation quality according to the Ottawa scale, patient tolerability, compliance, incidence of adverse events, sleep quality, and polyp/adenoma detection rate. Results: Among the 196 patients analyzed (mean age, 55.3 years; $50.3 \%$ men), 97 received the same-day regimen of 2 sachets of picosulfate (group A) and 99 received the $4 \mathrm{~L}$ split-dose PEG regimen (group B). The Ottawa score of the total colon was $4.05 \pm 1.56$ in group A and $3.80 \pm 1.55$ in group B $(P=0.255)$. The proportion of patients having adequate bowel preparation in the same-day picosulfate group (61.5\%) was slightly less than the $4 \mathrm{~L}$ PEG group (71.3\%); however, the difference was not statistically significant $(P=0.133)$. Tolerability of the group A regimen was superior to that of the group B regimen $(P<0.000)$. The same-day picosulfate regimen was associated with fewer adverse events, such as abdominal bloating $(P=0.037)$ and better sleep quality $(P<0.000)$. Conclusions: The same-day picosulfate regimen and the $4 \mathrm{~L}$ split-dose PEG regimen had similar efficacy in bowel preparation for afternoon colonoscopy. However, the sameday picosulfate regimen was easier to administer, produced fewer adverse events, and enabled better sleep quality. (Intest Res 2014;12:53-59)

Key Words: Colonoscopy; Sodium picosulfate; Polyethylene glycol

\section{INTRODUCTION}

Colonoscopy is the gold standard for the early detection and prevention of colorectal cancer. ${ }^{1,2}$ The increasing use of screening colonoscopy is crucial for the reduction of the incidence of colorectal cancer. ${ }^{3}$ Colon cleansing before a colonoscopy is essential for an adequate mucosal examination.

Received May 14, 2013. Revised October 1, 2013. Accepted October 2, 2013. Correspondence to Tae Oh Kim, Department of Internal Medicine, Haeundae Paik Hospital, Inje University College of Medicine, 875 Haeundae-ro,

Haeundae-gu, Busan 612-030, Korea. Tel: +82-51-797-0658, Fax: +82-51-

797-0298, E-mail: kto0440@paik.ac.kr

Financial support: None. Conflict of interest: None.
Inadequate preparation of the colon and rectum can lead to cancelled procedures, increased procedural times, and missed detection of lesions. ${ }^{4-6}$ In our experience, poor patient tolerance of the regimen involving administration of $4 \mathrm{~L}$ of polyethylene glycol (PEG) is frequently related to the ingestion of a large amount of fluid. Sodium phosphate is a lowvolume laxative that is superior to a single $4 \mathrm{~L}$ volume of $\mathrm{PEG}$ in terms of patient acceptance and cleansing quality. ${ }^{7}$ However, sodium phosphate has been associated with electrolyte disturbance, congestive heart failure, and renal failure. ${ }^{8,9}$ These problems have led to the introduction of similar preparations designed for more acceptable taste, requirement of a lower volume, and fewer adverse events. Sodium picosulfate is a low-volume bowel cleansing agent dispensed in powder

(c) Copyright 2014. Korean Association for the Study of Intestinal Diseases. All rights reserved.

This is an Open Access article distributed under the terms of the Creative Commons Attribution Non-Commercial License (http://creativecommons.org/licenses/by-nc/3.0)

which permits unrestricted non-commercial use, distribution, and reproduction in any medium, provided the original work is properly cited. 
form and dissolved in $150 \mathrm{~mL}$ of water prior to administration. The cleaning agent is composed of sodium picosulfate, magnesium oxide, and citric acid. ${ }^{10-12}$ The conventional bowel preparation regimen for afternoon colonoscopy involves a split-dose, in which the oral agent is administered the day before and on the morning of the procedure. The regimen involves a lengthy time gap between the bowel preparation and the procedure, which can lead to the accumulation of intestinal secretions and deterioration in mucosal cleansing. ${ }^{13}$ Furthermore, an often-overlooked factor for colonoscopy is the importance of the patient's quality of life. The split-dose approach requires patient involvement the afternoon of the day preceding the procedure and on the day of the procedure. This restricts the patients in performing other activities for a prolonged time and may lead to diarrhea that occurs in the night and disrupts sleep. Recently, a same-day bowel cleansing regimen using sodium picosulfate for afternoon colonoscopy has been reported to be superior to a split-dose regimen. ${ }^{13}$ Sodium picosulfate, administered in a split dose, reportedly provided superior bowel cleansing compared with the use of a standard 4 L PEG solution as a bowel preparation for colonoscopy. ${ }^{14}$ However, to our knowledge, no large randomized controlled trial has been conducted to compare conventional split-dose PEG with same-day picosulfate for afternoon colonoscopy. We hypothesized that the sameday sodium picosulfate bowel preparation and conventional split-dose PEG bowel preparation regimen would be similar in their quality of bowel preparation, and the present study was performed to assess this hypothesis.

\section{METHODS}

\section{Design of the Study and Patients}

This was an investigator-blinded, prospective, randomized, controlled study of outpatients. All patients aged $\geq 18$ years undergoing routine elective colonoscopy were invited to participate. We investigated the medical history of all patents by medical chart review and questionnaire. Patients with $\geq 1$ of the following conditions were excluded: pregnancy; breast feeding; prior history of surgical large bowel resection; severe constipation; consumption of antidiarrheal agents; severe medical conditions including severe cardiac disorders (recent myocardial infarction, unstable angina, congestive heart failure), hepatic failure, and renal failure (creatinine $\geq 3.0 \mathrm{mg} /$ $\mathrm{dL}$ [normal range, $0.8-1.4 \mathrm{mg} / \mathrm{dL}]$ ); significant gastric paresis or gastric outlet obstruction or ileus; known or suspected bowel obstruction or perforation; allergy to PEG or picosulfate; drug addiction or major psychiatric illness; or refusal to participate. All patients provided written informed consent. We prospectively enrolled consecutive outpatients with appropriate indications for colonoscopy. This study was conducted at a single university hospital, (Haeundae Paik Hospital) in Busan, Korea, between September and November
2012, and was approved by the hospital's institutional review board and registered in the clinical trial database at www. clinicaltrials.gov (NCT01685970).

\section{Bowel Preparation Methods}

All patients in both groups were instructed to ingest a lowresidue diet for 3 days prior to the colonoscopy and received information regarding unacceptable foods, which included vegetables containing high dietary fiber (e.g., kimchi, cabbage and green onion), seed-containing fruits (e.g., grape, water melon, and oriental melon), grains containing high dietary fiber (e.g., brown rice, black rice, and rice with mixed grains), seaweed (e.g., laver and sea mustard), dry beans, and nuts. For the same-day regimen (group A), patients were asked to ingest a sachet of sodium picosulfate (PICOLIGHT ${ }^{\circledR}$, Pharmbio Korea Co., Ltd., Seoul, Korea) at 6:00 and 10:00 AM for afternoon colonoscopy. Patients were instructed to pour $150 \mathrm{~mL}$ of water into each sachet and drink the entire volume at 6:00 and 10:00 AM, and then drink more than $1 \mathrm{~L}$ of water until 3 hours before the afternoon colonoscopy. For the split-dose regimen (group B), patients were instructed to ingest 2 L of PEG solution (Colyte ${ }^{\circledR}$, Taejoon Pharm. Co., Ltd., Seoul, Korea; 236 g PEG, $22.74 \mathrm{~g} \mathrm{Na}_{2} \mathrm{SO}_{4}$, $6.74 \mathrm{~g} \mathrm{NaHCO}_{3}$, $5.86 \mathrm{~g} \mathrm{NaCl}$, and $2.97 \mathrm{~g} \mathrm{KCl}$ ) at 6:00 PM on the day before the procedure, to begin the ingestion the remaining $2 \mathrm{~L}$ of PEG 5 hours prior to the procedure, and to finish the ingestion of the dose 3 hours prior to the colonoscopy on the day of the procedure.

\section{Randomization and Blinding}

A total of 203 patients were recruited, and 197 patients were enrolled after the assessment of the appropriate indications and after ruling out any contraindication for the colonoscopy. We generated a randomization schedule using the resources on a website (www.randomization.com) by the method of randomly permuted blocks. An investigator who was not involved in the colonoscopy conducted random assignments of patients to the same-day group (group A) or to the split-dose group (group B) according to the randomization schedule. The investigator who planned the randomization instructed the patients not to disclose their bowel preparation methods to the assisting nurses and colonoscopists. Colonoscopists did not have any information about the bowel preparation methods at the time of the procedure and remained blinded for the duration of the study. They only scored the bowel preparation scale and recorded colonoscopic data, which were sent to an investigator in charge of data processing. Colonoscopists were not involved in data collection or statistical analysis. 


\section{Assessment of Primary Outcome: Bowel Preparation Quality}

All study procedures were performed by 3 expert colonoscopists (TO Kim, JH Park, and EH Seo). All 3 had experience with more than 2,000 colonoscopy cases, had been involved in several bowel preparation studies using the Ottawa scale, and were qualified to score the bowel preparation scale. Bowel cleansing was evaluated by these 3 blinded colonoscopists using the Ottawa bowel preparation scale. ${ }^{15}$ This scale assesses cleanliness and fluid volume separately. Cleanliness was assessed for the right colon (cecum, ascending), middle colon (transverse, descending), and the rectosigmoid colon; each colon section was individually rated from 0 to 4 (no liquid: 0; minimal liquid, no suctioning required: 1 ; suction required to view the mucosa: 2; wash and suction: 3 ; solid stool, not washable: 4). Fluid quantity was rated from 0 to 2 for the entire colon (minimal: 0 ; moderate: 1 ; large: 2 ). The Ottawa scale ranges from 0 (perfect) to 14 (solid stool in each colon segment and copious fluid i.e., a completely unprepared colon). Investigators participating in the study were instructed on the manner in which the quality of bowel preparation could be assessed according to this scale. Prior to study initiation, the 3 colonoscopists performed calibration exercises involving 20 colonoscopies. The interclass correlation coefficient (ICC), $\kappa$, was estimated as 0.95 , indicating a very high level of inter-rater consistency. A complete colonoscopy was defined as a successful cecal intubation by visualization of the appendiceal orifice.

\section{Assessment of Secondary Outcomes: Patient Tolera- bility, Adverse Events, Sleep, and Polyp Detection}

Patients were asked to complete a questionnaire immediately before the procedure to evaluate patient tolerability, adverse events, and sleep. Patient tolerability was measured by the ease of drinking the PEG and picosulfate solutions, and of following the dietary instructions according to a 10-cm visual analogue scale (VAS) ("very easy: 0 " on the left end to "very difficult: 10 " on the right end). Adverse events reported by patients included abdominal pain or bloating, vomiting, nausea, and headache/dizziness. Sleep quantity was evaluated by patients' estimation of whether their hours of sleep for the night before the colonoscopy were more than $80 \%$ of their usual sleep duration or not. The number of polyps and adenomas detected was determined.

\section{Sample Size Calculation and Statistical Analyses}

The sample-size calculation was based on the comparison of the quality of preparation scores between the 2 groups. The analysis of the primary endpoint of preparation quality involved the evaluation of the non-inferiority of administration of 2 sachets of the picosulfate group compared to the
4 L PEG regimen with a pre-specified margin. In previous studies, the mean total Ottawa score of bowel preparation was approximately 5.0 with a standard deviation of 2.0. We hypothesized that a difference of $<15 \%$ in the mean total Ottawa score between the 2 groups could be defined as noninferior, and the non-inferiority margin was set at 0.75 . To confirm the non-inferiority, the absolute value of the lower limit of the 95\% confidence interval of the mean total score difference of the picosulfate group compared to the 4 L PEG regimen should not be more than 0.75 . With a power of $80 \%$ and an alpha-error of 5\%, the sample size was estimated to be 89 patients in each group using the Power Analysis and Sample Size Software (PASS) program. We assumed a dropout rate of $10 \%$, and planned to enroll a total of 196 patients. SAS version 9.1 (SAS Institute, Cary, NC, USA) was used for data analyses. Continuous variables are reported as the mean $\pm \mathrm{SD}$ and categorical variables are reported as percentages. A 2-sided $t$-test was used to compare the means of continuous variables in the 2 groups and a Pearson $\chi^{2}$ test was used to compare the categorical variables. A $P$-value of $<0.05$ was considered statistically significant.

\section{RESULTS}

\section{Patient Characteristics}

A total of 203 consecutive patients referred for outpatient colonoscopy were assessed for eligibility. Six patients were excluded, including 2 with chronic kidney disease (creati-

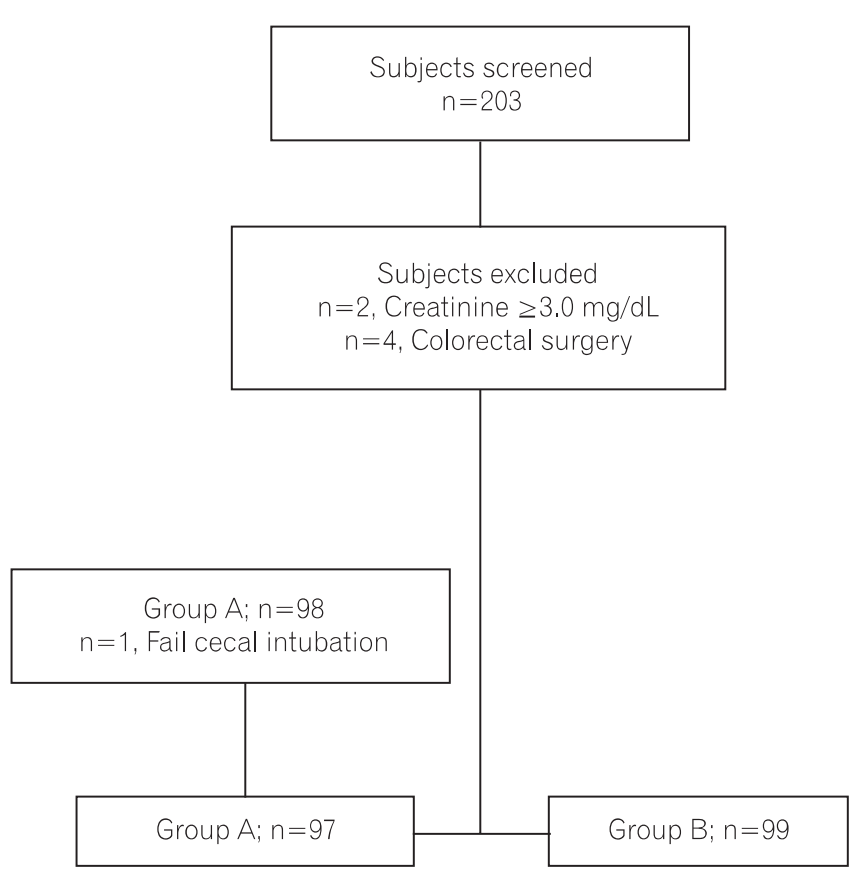

Fig. 1. Assignment of subjects into groups. Group A: 2 sachets of sodium picosulfate. Group B: $4 \mathrm{~L}$ of polyethylene glycol. 
nine $\geq 3.0 \mathrm{mg} / \mathrm{dL}$ ) and 4 with a history of previous colorectal surgery. The 197 patients were randomly assigned to receive one of the 2 preparation methods. One patient in the picosulfate group was not eligible to complete cecal intubation due to loop formation. A total of 196 patients (97 in the picosulfate group and 99 in the 4 L PEG group) were included in the final analysis (Fig. 1). There were no significant differences between the 2 groups with regard to the patient demographics including age, sex, or average BMI (Table 1).

\section{Primary End-point: Bowel Preparation Quality}

The mean Ottawa scale scores for each group are shown in Table 2. The Ottawa score of the total colon was $4.05 \pm 1.56$ in the same-day picosulfate group and $3.80 \pm 1.55$ in the $4 \mathrm{~L}$ PEG group $(P=0.255)$. An adequate bowel preparation was defined as an Ottawa score of 0 to $5 .^{16}$ The proportion of patients having adequate bowel preparation in the same-day picosulfate group (61.5\%) was slightly less than the 4 L PEG group (71.3\%), but the difference was not statistically significant $(P=0.133)$. The scores of the middle and rectosigmoid colon segments were not significantly different between the 2 groups. The score of the right colon was $1.65 \pm 0.70$ in the same-day picosulfate group (group A) and $1.43 \pm 0.77$ in the 4 L PEG group (group B) $(P=0.046)$. The score of fluid volume was $0.35 \pm 0.48$ in group A and $0.56 \pm 0.54$ in group $B(P=0.05)$. The right segments were slightly less clear in group $A$, but

Table 1. Baseline Characteristics of the Patients

\begin{tabular}{lccc}
\hline \multicolumn{1}{c}{ Characteristic } & $\begin{array}{c}\text { Group A } \\
\mathbf{n = 9 7}\end{array}$ & $\begin{array}{c}\text { Group B } \\
\mathbf{n = 9 9}\end{array}$ & P-value \\
\hline Age (yr) & $56.8 \pm 11.9$ & $53.2 \pm 13.3$ & 0.108 \\
Male/Female & $41 / 56$ & $54 / 45$ & 0.088 \\
BMI $\left(\mathrm{kg} / \mathrm{m}^{2}\right)$ & $23.5 \pm 2.8$ & $23.4 \pm 2.7$ & 0.686 \\
Experience with colonoscopy & $51(52)$ & $39(39.4)$ & 0.087 \\
Family history of colon cancer & $5(5.1)$ & $5(5.1)$ & 1.000 \\
Previous surgical history & $28(28.6)$ & $17(17.2)$ & 0.063 \\
(abdominopelvic surgery) & & & \\
Past medical history & & & 0.798 \\
$\quad$ Diabetes & $2(2)$ & $2(2)$ & \\
Hypertension & $21(21.4)$ & $21(21.2)$ & \\
Cerebral vascular disease & $1(1)$ & $1(1)$ & \\
Thyroid disease & $1(1)$ & $1(1)$ & \\
Ischemic heart disease & $2(2)$ & $1(1)$ & \\
Other & $6(6.1)$ & $3(3)$ & \\
\hline
\end{tabular}

Values are presented as means \pm SD or $n(\%)$.

Group A: 2 sachets of sodium picosulfate.

Group B: $4 \mathrm{~L}$ of polyethylene glycol.

Other: Hepatitis (Alcoholic; toxic; or viral B or C) and prior history of tuberculosis. the quantity of fluid was less than that in group B. The bowel preparation quality of the same-day group was not inferior to that of the 4 L PEG group, based on the assumption of a prespecified non-inferiority margin of the mean total Ottawa scale score difference of 0.75 .

\section{Secondary End-point: Number of Polyps or Adenomas}

Table 3 shows the colonoscopy data of the 2 groups. The polyp detection rate was slightly higher in the $4 \mathrm{~L}$ PEG group (50.5\% vs. $38.5 \%, P=0.113$ ), indicating a trend toward slightly superior detection in this group. Adenoma detection rates were similar between the 2 groups (34.3\% for the PEG group Vs. $31.3 \%$ for the same-day group, $P=0.651$ ). A numerical trend was observed for the slightly superior polyp detection rate in the $4 \mathrm{~L}$ PEG group. However, no significant difference in the adenoma detection rate was observed between the 2 groups.

\section{Secondary End-point: Patient Tolerability, Adverse Events, and Sleep Disturbance}

The data collected from the patient questionnaire about the tolerability of bowel preparation are summarized in Table

Table 2. Outcome of Bowel Cleansing according to the SubCategories of the Ottawa Bowel Scale

\begin{tabular}{|c|c|c|c|}
\hline Ottawa scale score & $\begin{array}{c}\text { Group A } \\
n=97\end{array}$ & $\begin{array}{c}\text { Group B } \\
n=99\end{array}$ & $P$-value \\
\hline Right colon & $1.65 \pm 0.70$ & $1.43 \pm 0.77$ & 0.046 \\
\hline Middle colon & $1.18 \pm 0.50$ & $1.08 \pm 0.42$ & 0.154 \\
\hline Rectosigmoid colon & $0.88 \pm 0.60$ & $0.75 \pm 0.50$ & 0.104 \\
\hline Fluid quantity & $0.35 \pm 0.48$ & $0.56 \pm 0.54$ & 0.005 \\
\hline Total score & $4.05 \pm 1.56$ & $3.80 \pm 1.55$ & 0.255 \\
\hline $\begin{array}{l}\text { Adequate bowel } \\
\text { preparation score }(<5)\end{array}$ & $59(61.5)$ & 71 (71.3) & 0.133 \\
\hline $\begin{array}{l}\text { Inadequate bowel prep. } \\
\text { score }(\geq 5)\end{array}$ & $37(38.5)$ & $28(28.3)$ & \\
\hline
\end{tabular}

Values are presented as means \pm SD or $n(\%)$.

Group A: 2 sachets of sodium picosulfate.

Group B: $4 \mathrm{~L}$ of polyethylene glycol.

Table 3. Status of All Polyps Detected in Patients Who Received Bowel Preparations

\begin{tabular}{|c|c|c|c|}
\hline & $\begin{array}{c}\text { Group A } \\
n=97(\%)\end{array}$ & $\begin{array}{c}\text { Group B } \\
n=99(\%)\end{array}$ & $P$-value \\
\hline Any polyp & $37(38.5)$ & $50(50.5)$ & 0.11 \\
\hline Multiple polyps ( $\geq 3$ ) & $11(11.3)$ & $12(12.1)$ & 1.00 \\
\hline Any adenoma & $30(31.3)$ & $34(34.3)$ & 0.65 \\
\hline
\end{tabular}


Table 4. Tolerability of the Bowel Preparations

\begin{tabular}{lccc}
\hline & Group A & Group B & $\boldsymbol{P}$-value \\
\hline Ease of taking solutions & $2.17 \pm 2.23$ & $5.97 \pm 2.42$ & $<0.001$ \\
Ease of restricted diet & $4.02 \pm 2.82$ & $4.04 \pm 2.36$ & 0.35 \\
\hline
\end{tabular}

Values are presented as means \pm standard deviation.

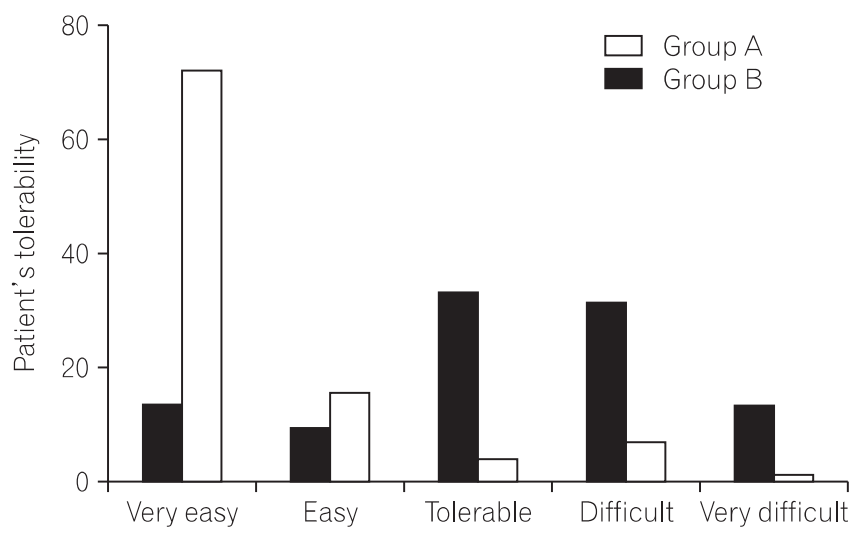

Fig. 2. Patient's ability to tolerate the bowel preparation. The tolerability of same-day picosulfate was superior to split-dose PEG $(P<0.001)$.

4 and Fig. 2. None of the patients failed to ingest the bowel preparation solution. Ninety-seven patients in group A and 99 patients in group B ingested $>90 \%$ of the total bowel preparation solution $(P=0.721)$. Two sachets of picosulfate were easier to ingest (VAS for difficulty, $2.17 \pm 2.23$ ) than the $4 \mathrm{~L}$ volume of PEG (VAS for difficulty, $5.95 \pm 2.40 ; P<0.001$ ) (Table 4 ). The proportion of patients expressing easiness of bowel preparation was higher in the same-day picosulfate group than the 4 L PEG group (VAS for difficulty, 1-2 [i.e., very easy]: $71 \%$ of those in the same-day picosulfate group vs. $13 \%$ in the PEG group; $P<0.001$ ) (Fig. 2). Adverse events before the procedure were collected via a questionnaire and assessed according to the bowel preparation regimen (Table 5). No severe adverse events were reported in any of the cases. The overall incidence of adverse events was lower in the sameday picosulfate group $(20.4 \%$ in the same-day picosulfate group and $34.3 \%$ in the $4 \mathrm{~L}$ PEG group, $P=0.037$ ). There were more frequent adverse events, such as abdominal pain, headache, dizziness, nausea, abdominal bloating, and vomiting, in the 4 L PEG group (Table 5). In addition, patients in the sameday picosulfate group had better sleep quality during the night prior to the colonoscopy as compared to patients in the 4 L PEG group. The proportion of patients who slept at least $80 \%$ of their usual sleeping hours was significantly larger in the same-day picosulfate group $(87.8 \%)$ than in the $4 \mathrm{~L} \mathrm{PEG}$ group $(56.4 \%)(P<0.001)$.
Table 5. Adverse Events according to the Bowel Preparation Regimen

\begin{tabular}{lccr}
\hline & $\begin{array}{c}\text { Group A } \\
\mathbf{n = 9 7}\end{array}$ & $\begin{array}{c}\text { Group B } \\
\mathbf{n = 9 9}\end{array}$ & P-value \\
\hline $\begin{array}{l}\text { Any side effect (except sleep } \\
\text { disturbance) }\end{array}$ & $20(20.4)$ & $34(34.3)$ & 0.037 \\
Abdominal pain & $4(4.1)$ & $7(7.1)$ & 0.537 \\
Abdominal distension & $4(4.1)$ & $16(16.2)$ & 0.008 \\
Vomiting & $1(1)$ & $9(9.1)$ & 0.018 \\
Nausea & $17(17.3)$ & $11(11.1)$ & 0.227 \\
Headache/dizziness & $5(5.1)$ & $7(7.1)$ & 0.767 \\
Interruption of sleep & $12(12.2)$ & $44(44.4)$ & $<0.001$ \\
\hline Values are presented as $n(\%)$ & & &
\end{tabular}

\section{DISCUSSION}

This was a prospective, randomized study designed to evaluate the efficacy and tolerability of same-day administration of 2 sachets of picosulfate for afternoon colonoscopy compared with the standard split-dose $4 \mathrm{~L}$ volume of PEG. Recently, Gaius et al. reported that a regimen involving 2 sachets of picosulfate bowel preparation was superior to a conventional split-dose regimen of 3 sachets of sodium picosulfate. $^{13}$ The present regimen enables the patient to perform normal activities on the previous day and evening and does not interrupt sleep on the night before the procedure. PEG is widely used as a bowel preparation for colonoscopy. The regimen has no significant effect on intra- and extravascular volume or on the levels of serum electrolytes. ${ }^{17}$ Despite its proven efficacy and safety, this laxative is difficult for many patients to tolerate. The $4 \mathrm{~L}$ volume of PEG that is required for effective lavage can be difficult to consume. ${ }^{14}$ Furthermore, the dose ingested on the night before the procedure often disrupts sleep. Conventional bowel preparation for afternoon colonoscopy cases also involves a split-dose regimen. However, this approach can result a significant time gap between bowel preparation and the procedure, allowing accumulation of intestinal secretions and deterioration of mucosal cleansing. ${ }^{13}$ An often-overlooked, yet important, factor in colonoscopy is the patient's quality of life. Several recent studies have reported that 1 day bowel preparation for colonoscopy is beneficial because the timing of bowel preparation is vital. ${ }^{18-22}$ We tested whether the same-day bowel preparation was suitable for afternoon colonoscopy. This study showed that patient tolerability was better for the same-day bowel preparation and that the bowel cleansing was not inferior to a splitdose of PEG over 2 days.

Picosulfate increases the frequency and force of peristalsis, resulting in electrolyte and water retention in the colon, which can lead to hyponatremia, dehydration, and other electrolyte imbalances. However, there have been no reports of clinically significant adverse events. ${ }^{10,12,14,18,23}$ Picosulfate is 
a low-volume lavage solution that has a better taste than PEG. Low-volume lavage solution, good taste, and same-day bowel preparation improved tolerability and led to fewer adverse events in this study. Both groups had no complications such as perforation or hemorrhage during or after colonoscopy.

Our study had 2 limitations. First, adverse-effect data was obtained through a questionnaire. The same-day picosulfate bowel preparation group self-reported fewer adverse events, such as nausea, abdominal pain, and vomiting compared with the group undergoing conventional bowel preparation using a $4 \mathrm{~L}$ split dose of PEG. These adverse events have also been reported in other studies. ${ }^{18,21}$ There were no severe complications, such as hemodynamic instability, after taking picosulfate. We believe that fewer adverse events equates to less disturbance of the serum biochemistry. However, rigorous evaluation of the levels of electrolytes and renal function, among other parameters, is required. Second, although all patients in the 2 picosulfate sachets group ingested both sachets, the volume of water consumed varied from $0.5 \mathrm{~L}$ to $3 \mathrm{~L}$. The same-day group was instructed to ingest over $1 \mathrm{~L}$ of water up to 3 hours before the procedure. Patients ingesting less water exhibited poor bowel preparation, such as more turbid mucosa. These data are not shown because we had not initially planned to include the volume of water ingested in our questionnaire. The relationship between the ingested volume of water and the quality of bowel preparation needs to be studied. Moreover, in the present study, we used the Ottawa scoring system for bowel cleansing. It assesses the colonic segments individually and colonic fluid overall, and provides a summary score for the entire colon. In our study, split-dose PEG and picosulfate had similar cleansing efficacy in terms of the total Ottawa score. However, the right colon score in the picosulfate group was slightly higher than that in the splitdose PEG group ( 1.65 vs. 1.43, $P=0.046$ ). The fluid quantity ingested in the picosulfate group was less than that in the splitdose PEG group ( 0.36 vs. 0.56, $P=0.005)$. Therefore, the total values are similar in the 2 groups. This indicates that mucosal cleansing using the picosulfate method is slightly less efficient than the split-dose PEG approach. In contrast, the polyp detection and adenoma detection rates did not differ significantly between the 2 groups. In conclusion, a regimen involving same-day administration of 2 sachets of picosulfate was as effective as a split-dose 4 LPEG regimen, but was associated with fewer adverse events and better patient tolerability. The same-day administration of 2 sachets of picosulfate bowel preparation saves time and does not disturb sleep during the previous night. This same-day bowel preparation for afternoon colonoscopy will be preferred by many patients and examiners. However, for routine use of this regimen for afternoon colonoscopy, multicenter, randomized, controlled trials that show effective mucosal cleansing, improved tolerability, and less complications are required.

\section{REFERENCES}

1. Lieberman DA, Weiss DG, Bond JH, Ahnen DJ, Garewal H, Chejfec G. Use of colonoscopy to screen asymptomatic adults for colorectal cancer. Veterans Affairs Cooperative Study Group 380. N Engl J Med 2000;343:162-168.

2. Winawer SJ, Zauber AG, Ho MN, et al. Prevention of colorectal cancer by colonoscopic polypectomy. The National Polyp Study Workgroup. N Engl J Med 1993;329:1977-1981.

3. Harewood GC, Wiersema MJ, Melton LJ 3rd. A prospective, controlled assessment of factors influencing acceptance of screening colonoscopy. Am J Gastroenterol 2002;97:3186-3194.

4. Chiu HM, Lin JT, Wang HP, Lee YC, Wu MS. The impact of colon preparation timing on colonoscopic detection of colorectal neoplasms--a prospective endoscopist-blinded randomized trial. Am J Gastroenterol 2006;101:2719-2725.

5. Froehlich F, Wietlisbach V, Gonvers JJ, Burnand B, Vader JP. Impact of colonic cleansing on quality and diagnostic yield of colonoscopy: the European Panel of Appropriateness of Gastrointestinal Endoscopy European multicenter study. Gastrointest Endosc 2005;61:378-384.

6. Rex DK. Maximizing detection of adenomas and cancers during colonoscopy. Am J Gastroenterol 2006;101:2866-2877.

7. Vanner SJ, MacDonald PH, Paterson WG, Prentice RS, Da Costa LR, Beck IT. A randomized prospective trial comparing oral sodium phosphate with standard polyethylene glycol-based lavage solution (Golytely) in the preparation of patients for colonoscopy. Am J Gastroenterol 1990;85:422-427.

8. Hookey LC, Depew WT, Vanner S. The safety profile of oral sodium phosphate for colonic cleansing before colonoscopy in adults. Gastrointest Endosc 2002;56:895-902.

9. Ahmed M, Raval P, Buganza G. Oral sodium phosphate catharsis and acute renal failure. Am J Gastroenterol 1996;91:1261-1262.

10. Hoy SM, Scott LJ, Wagstaff AJ. Sodium picosulfate/magnesium citrate: a review of its use as a colorectal cleanser. Drugs 2009;69: 123-136.

11. Clark LE, Dipalma JA. Safety issues regarding colonic cleansing for diagnostic and surgical procedures. Drug Saf 2004;27:12351242.

12. Di Palma JA, Buckley SE, Warner BA, Culpepper RM. Biochemical effects of oral sodium phosphate. Dig Dis Sci 1996;41:749753.

13. Longcroft-Wheaton G, Bhandari P. Same-day bowel cleansing regimen is superior to a split-dose regimen over 2 days for afternoon colonoscopy: results from a large prospective series. J Clin Gastroenterol 2012;46:57-61.

14. Rex DK, Di Palma JA, Rodriguez R, McGowan J, Cleveland M. A randomized clinical study comparing reduced-volume oral sulfate solution with standard 4-liter sulfate-free electrolyte lavage solution as preparation for colonoscopy. Gastrointest Endosc 2010;72:328-336.

15. Rostom A, Jolicoeur E. Validation of a new scale for the assessment of bowel preparation quality. Gastrointest Endosc 2004;59: 482-486. 
16. Seo EH, Kim TO, Park MJ, et al. Optimal preparation-to-colonoscopy interval in split-dose PEG bowel preparation determines satisfactory bowel preparation quality: an observational prospective study. Gastrointest Endosc 2012;75:583-590.

17. Seo EH, Kim TO, Kim TG, et al. Efficacy and tolerability of splitdose PEG compared with split-dose aqueous sodium phosphate for outpatient colonoscopy: a randomized, controlled trial. Dig Dis Sci 2011;56:2963-2971.

18. Di Palma JA, Rodriguez R, McGowan J, Cleveland M. A randomized clinical study evaluating the safety and efficacy of a new, reduced-volume, oral sulfate colon-cleansing preparation for colonoscopy. Am J Gastroenterol 2009;104:2275-2284.

19. Poon CM, Lee DW, Mak SK, et al. Two liters of polyethylene glycol-electrolyte lavage solution versus sodium phosphate as bowel cleansing regimen for colonoscopy: a prospective randomized controlled trial. Endoscopy 2002;34:560-563.
20. Eun CS, Han DS, Hyun YS, et al. The timing of bowel preparation is more important than the timing of colonoscopy in determining the quality of bowel cleansing. Dig Dis Sci 2011;56:539-544.

21. Hosoe N, Nakashita M, Imaeda H, et al. Comparison of patient acceptance of sodium phosphate versus polyethylene glycol plus sodium picosulfate for colon cleansing in Japanese. J Gastroenterol Hepatol 2012;27:1617-1622.

22. Parra-Blanco A, Nicolas-Perez D, Gimeno-Garcia A, et al. The timing of bowel preparation before colonoscopy determines the quality of cleansing, and is a significant factor contributing to the detection of flat lesions: a randomized study. World J Gastroenterol 2006;12:6161-6166.

23. Flemming JA, Vanner SJ, Hookey LC. Split-dose picosulfate, magnesium oxide, and citric acid solution markedly enhances colon cleansing before colonoscopy: a randomized, controlled trial. Gastrointest Endosc 2012;75:537-544. 